\title{
Case report: Silicone is not fun in the sun
}

\author{
Arthur E Brawer* \\ Department of Medicine, Division of Rheumatology, Monmouth Medical Center, USA
}

\begin{abstract}
A 34-year-old white female presented with a multisystem illness five years after undergoing cosmetic breast augmentation with silicone gel-filled breast implants. Her most prominent clinical features were repetitive photosensitivity and persistent polyarthritis, both occurring for the first time six months after her surgery. The differential diagnoses of her treating physicians did not include device-related toxicity because they were unaware of the reality and verifiable manifestations of silicone-induced disease.
\end{abstract}

\section{Case report}

A healthy and athletically active 29 -year-old white female, on no medications, underwent cosmetic breast enhancement with the insertion of silicone gel-filled breast implants. Six months after her surgery, while vacationing in the Caribbean, she developed a raised, erythematous pruritic rash on the anterior and posterior trunk after twenty minutes of continuous sun exposure (Figure 1). For six consecutive years prior to her breast augmentation she had vacationed in the sun on the same island without incident. The rash was accompanied by pain, stiffness and swelling in her hands, wrists, neck, knees, ankles and feet. Although the rash resolved after ten days of sun avoidance, the joint symptoms persisted. There was no immediate history of any insect bites, fever, headaches, lymphadenopathy, sore throat, gastrointestinal complaints or lower respiratory symptoms.

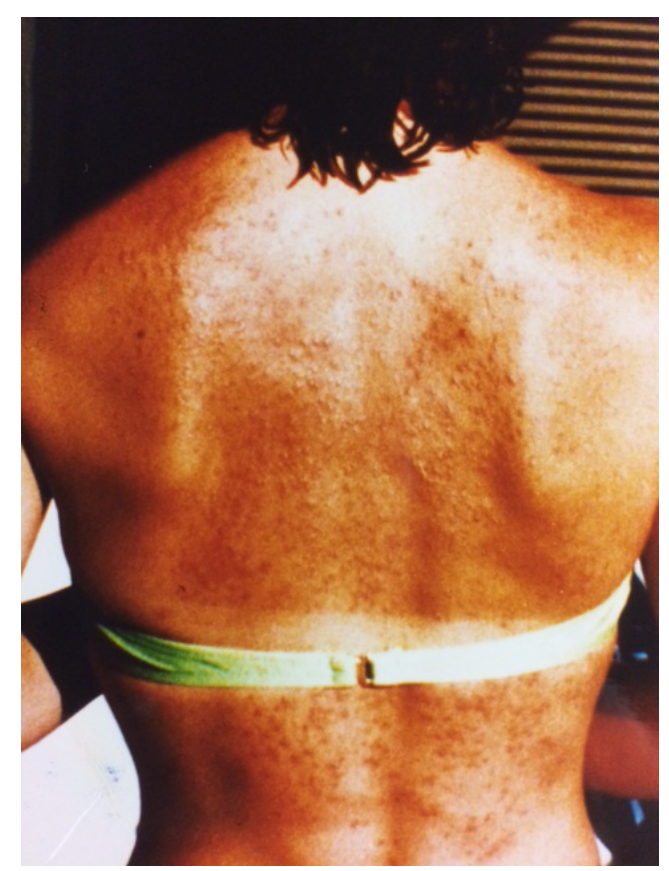

Figure 1. Six months after her surgery, while vacationing in the Caribbean, she developed a raised, erythematous pruritic rash on the anterior and posterior trunk after twenty minutes of continuous sun exposure
One month later she developed fatigue, one hour of morning stiffness, myalgias in all four extremities, paresthesias in her feet and hands, and the first of six dental extractions because of newly developing and progressive periodontal disease. Over the next four years she developed dry eyes, night sweats, scalp hair loss, easy bruisability, chest pains, vague abdominal pains, telangiectasias on the upper anterior chest, cognitive dysfunction (memory lapses, and problems with word recall and name recall), repetitive episodes of identical photosensitivity after brief sun exposure, pruritis even without reappearance of her rash, and multiple enlarged axillary lymph nodes bilaterally. During this interval she underwent multiple rheumatology and infectious disease evaluations, accompanied by exhaustive laboratory testing, all of which failed to yield a specific diagnosis. In particular, repetitive antinuclear antibody tests and infectious serologies were negative. Treatment with hydroxychloroquine and oral corticosteroids afforded no improvement in her condition. Additional physical examination findings five years after her augmentation surgery included normal proximal muscle strength, no tight skin, tenderness to palpation of multiple anterolateral ribs, a $20 \mathrm{cc}$ effusion in the left knee, swelling in her hands and wrists, and a Schirmer test yielding $5 \mathrm{~mm}$ of tear formation after five minutes. Minor salivary gland biopsy was normal. Arthrocentesis of 10cc yellow fluid from the left knee revealed only 100 white cells, a good mucin clot, no crystals, and a negative culture. Chest x-ray, mammography, EMG's and EKG were unremarkable. Biopsy of several axillary lymph nodes revealed chronic inflammation with foreign body giant cells, and clear droplets consistent with silicone. Shortly thereafter the patient underwent explantation of non-ruptured implants, accompanied by capsulectomies. During the next eleven months improvement and/or resolution of all symptoms and signs occurred.

${ }^{*}$ Correspondence to: Arthur E Brawer, M.D., Department of Medicine, Division of Rheumatology, Monmouth Medical Center, 300 Second Avenue, Long Branch, New Jersey 07740, USA, Tel: (732) 870-3133; Fax: (732) 222-0824; E-mail: arthurbrawer@optimum.net

Key words: silicone, breast implants, photosensitivity, toxicity

Received: October 19, 2018; Accepted: October 29, 2018; Published: October 31,2018 


\section{Discussion}

Over the past twelve years in the USA, following termination of the 14 and $1 / 2$ year-long FDA ban on silicone gel-filled breast implants (extending from April of 1992 to December of 2006), two-and-a-half million women have had these devices inserted into their bodies. Over the past four years more than 100,000 of these recipients have had them permanently removed to alleviate a multitude of ailments comparable to both (a) the systemic illness manifested by this patient, and (b) the illnesses manifested by 400,000 recipients 26 years ago [13]. Breast implants are now known to be a slow delivery system from day one, producing microdispersion of silicone to multiple distant sites beyond the local breast environment and axillary nodes [4]. This, in turn, initiates a multisystem disease whose subsequent chronological development simulates a dose-response curve [1,2]. Systemic silicone toxicity is a genuinely novel illness caused by over two dozen disruptions of the body's biochemistry, virtually none of which have anything to do with autoimmunity (and never did have anything to do with autoimmunity) [1-6]. Silicone breast implant illness is an evolving recurrent public health debacle that physicians should acknowledge and become familiar with.

\section{References}

1. Brawer AE (2017) Destiny rides again: the reappearance of silicone gel-filled breast implant toxicity. Lupus 26: 1060-1063. [Crossref]

2. Brawer AE (1996) Chronology of systemic disease development in 300 symptomatic recipients of silicone gel-filled breast implants. J Clean Technol Environ Toxicol Occup Med 5: 223-232.

3. Brawer AE (2012) Bones, groans, and silicone. Lupus 21: 1155-1157. [Crossref]

4. Brawer AE (2017) Mechanisms of breast implant toxicity: will the real ringmaster please stand up. Int Ann Med 1: 249.

5. Brawer AE (1998) Silicon and matrix macromolecules: new research opportunities for old diseases from analysis of potential mechanisms of breast implant toxicity. Med Hypotheses 51: 27-35. [Crossref]

6. Brawer AE (2017) Is silicone breast implant toxicity an extreme form of a more generalized toxicity adversely affecting the population as a whole?. Int Ann Med 1: 347 .

Copyright: $\odot 2018$ Brawer AE. This is an open-access article distributed under the terms of the Creative Commons Attribution License, which permits unrestricted use, distribution, and reproduction in any medium, provided the original author and source are credited. 